Draft version OCtober 30, 2018

Preprint typeset using $\mathrm{L}^{\mathrm{A}} \mathrm{T} \mathrm{E}$ X style emulateapj v. 11/10/09

\title{
BRIGHT POINTS IN THE QUIET SUN AS OBSERVED IN THE VISIBLE AND NEAR-UV BY THE BALLOON-BORNE OBSERVATORY SUNRISE
}

\author{
T. L. Riethmüller, ${ }^{1}$ S. K. Solanki, ${ }^{1,2}$ V. Martínez Pillet ${ }^{3}$ J. Hirzberger, ${ }^{1}$ A. Feller, ${ }^{1}$ J. A. Bonet ${ }^{3}$ N. Bello \\ González, ${ }^{4}$ M. Franz, ${ }^{4}$ M. Schüssler, ${ }^{1}$ P. Barthol, ${ }^{1}$ T. Berkefeld, ${ }^{4}$ J. C. del Toro Iniesta, ${ }^{5}$ V. Domingo, ${ }^{6}$ \\ A. Gandorfer, ${ }^{1}$ M. Knölker, ${ }^{7}$ \& W. Schmidt ${ }^{4}$ \\ ${ }^{1}$ Max-Planck-Institut für Sonnensystemforschung, Max-Planck-Str. 2, 37191 Katlenburg-Lindau, Germany; riethmueller@mps.mpg.de \\ ${ }^{2}$ School of Space Research, Kyung Hee University, Yongin, Gyeonggi, 446-701, Republic of Korea \\ ${ }^{3}$ Instituto de Astrofísica de Canarias, C/Via Láctea s/n, 38200 La Laguna, Tenerife, Spain \\ ${ }^{4}$ Kiepenheuer-Institut für Sonnenphysik, Schöneckstr. 6, 79104 Freiburg, Germany \\ ${ }^{5}$ Instituto de Astrofísica de Andalucía (CSIC), Apartado de Correos 3004, 18080 Granada, Spain \\ ${ }^{6}$ Grupo de Astronomía y Ciencias del Espacio, Universidad de Valencia, 46980 Paterna, Valencia, Spain \\ ${ }^{7}$ High Altitude Observatory, National Center for Atmospheric Research, P.O. Box 3000, Boulder, CO 80307-3000, USA
}

Draft version October 30, 2018

\begin{abstract}
Bright points (BPs) are manifestations of small magnetic elements in the solar photosphere. Their brightness contrast not only gives insight into the thermal state of the photosphere (and chromosphere) in magnetic elements, but also plays an important role in modulating the solar total and spectral irradiance. Here, we report on simultaneous high-resolution imaging and spectropolarimetric observations of BPs using SUNRISE balloon-borne observatory data of the quiet Sun at the disk center. BP contrasts have been measured between $214 \mathrm{~nm}$ and $525 \mathrm{~nm}$, including the first measurements at wavelengths below $388 \mathrm{~nm}$. The histograms of the BP peak brightness show a clear trend toward broader contrast distributions and higher mean contrasts at shorter wavelengths. At $214 \mathrm{~nm}$, we observe a peak brightness of up to five times the mean quiet-Sun value, the highest BP contrast so far observed. All BPs are associated with a magnetic signal, although in a number of cases it is surprisingly weak. Most of the BPs show only weak downflows, the mean value being $240 \mathrm{~m} \mathrm{~s}^{-1}$, but some display strong down- or upflows reaching a few $\mathrm{km} \mathrm{s}^{-1}$.

Subject headings: Sun: magnetic topology — Sun: photosphere — techniques: photometric — techniques: polarimetric - techniques: spectroscopic
\end{abstract}

\section{INTRODUCTION}

Bright points (BPs) are small-scale brightness enhancements located in the darker intergranular lanes. Dunn \& Zirker (1973) and Mehltretter (1974) were the first to describe these BPs in filter images taken in the far line wings of $\mathrm{H} \alpha$ and $\mathrm{Ca}$ II K, respectively. Common models consider BPs as radiative signatures of magnetic elements, which are often described by nearly vertical slender flux tubes or sheets (Deinzer et al. 1984; Solanki 1993). The increased magnetic pressure within the flux tube leads to its evacuation, and the lateral inflow of heat through the walls of the flux tube makes it hot and bright (Spruit 1976). Consequently, BPs are often used as tracers of magnetic elements.

The contrast of BPs relative to the average quietSun intensity is of interest since it provides insight into the structure and thermodynamics of magnetic elements. Furthermore, the excess brightness of magnetic elements is an important contributor to variations of the total solar irradiance (Solanki \& Fligge 2002). In the visible, the contrast of BPs is particularly high in wavelength regions that are dominated by absorption bands of temperature-sensitive molecules such as CH and CN (Muller \& Roudier 1984; Berger et al. 1995; Zakharov et al. 2005; Berger et al. 2007; Utz et al. 2009). We expect that their contrast is also large in the ultraviolet (UV), but they have never been studied at wavelengths shorter than $388 \mathrm{~nm}$.
In this work, we extend the study of BPs, in particular their contrasts, to the UV spectral range down to $214 \mathrm{~nm}$, using seeing-free images gathered by the balloon-borne 1$\mathrm{m}$ aperture SunRISE telescope. This is particularly important owing to the finding that irradiance changes below $400 \mathrm{~nm}$ produce over $60 \%$ of the variation of the total solar irradiance over the solar cycle (Krivova et al. 2006). The spectral and total irradiance variations are caused by the variation of magnetic flux at the solar surface, in particular in the form of small-scale magnetic elements (Krivova et al. 2003, 2006; Wenzler et al. 2006).

\section{OBSERVATIONS, DATA REDUCTION, AND ANALYSIS}

The data employed here were acquired during the 2009 June stratospheric flight of SunRISE. Technical details of the telescope are described by Barthol et al. (2010). Image stabilization, feature tracking, and correction of low-order wavefront aberrations were achieved by the gondola's pointing system in conjunction with a sixelement Shack-Hartmann correlating wavefront sensor (Berkefeld et al. 2010).

Observations in the near-ultraviolet spectral domain between $214 \mathrm{~nm}$ and $397 \mathrm{~nm}$ were acquired with the Sunrise Filter Imager (SuFI; Gandorfer et al. 2010). Simultaneously, the full Stokes magnetograph IMaX (Martínez Pillet et al. 2010) scanned the Fe I line at $525.02 \mathrm{~nm}$ (Landé factor $g=3$ ), hence providing kinematic and magnetic information. An overview of the collected data and a description of some of the observed 
TABLE 1

Exposure Times of the Used Time Series.

\begin{tabular}{llllc}
\hline $\begin{array}{l}\text { Central } \\
\text { Wavelength } \\
(\mathrm{nm})\end{array}$ & $\begin{array}{l}\text { FWHM } \\
\text { of Filter } \\
(\mathrm{nm})\end{array}$ & $\begin{array}{l}\text { 00:36-00:59 } \\
\text { Exp. Time } \\
(\mathrm{ms})\end{array}$ & $\begin{array}{l}\text { 01:31-02:00 } \\
\text { Exp. Time } \\
(\mathrm{ms})\end{array}$ & $\begin{array}{l}\text { 14:22-15:00 } \\
\text { Exp. Time } \\
(\mathrm{ms})\end{array}$ \\
\hline 214 & 10 & $\ldots$ & $\ldots$ & 30000 \\
300 & 5 & 500 & 500 & 250 \\
312 & 1.2 & 150 & 150 & 300 \\
388 & 0.8 & 80 & 80 & 150 \\
396.8 & 0.18 & 960 & 960 & 900 \\
525.02 & 0.0085 & 250 & 250 & 250 \\
\hline
\end{tabular}

phenomena is given by Solanki et al. (2010).

We use three time series recorded from 00:36 to 00:59 UT, 01:31 to 02:00 UT, and 14:22 to 15:00 UT on 2009 June 9 . At these times, the telescope pointed to quiet regions close to the disk center. The SuFI instrument recorded filtergrams centered at $397 \mathrm{~nm}$ (Ca II H), $388 \mathrm{~nm}$ (CN), $312 \mathrm{~nm}, 300 \mathrm{~nm}$, and $214 \mathrm{~nm}$, while IMaX was operated in its standard vector spectropolarimeter mode, i.e., full Stokes observations at five wavelength points with six accumulations. The five wavelength points were set to $\lambda-\lambda_{0}=-80,-40,+40,+80$, and $+227 \mathrm{~m} \AA$ relative to the center of the line. A summary of the filter widths (FWHM) and exposure times can be found in Table 11. The effective exposure time of an IMaX continuum image was $6 \mathrm{~s}$ (6 accumulations $\times 4$ modulation states $\times 250 \mathrm{~ms}$ ), while Dopplergrams need five wavelength points; i.e., their effective exposure time was 30 s (see Martínez Pillet et al. 2010, for more details).

At a typical flight altitude of around $36 \mathrm{~km}$, the $214 \mathrm{~nm}$ wavelength range was still strongly attenuated by the residual atmosphere of the Earth, so that an exposure time of $30 \mathrm{~s}$ was needed, even at the highest Sun elevations achieved during the flight. The $214 \mathrm{~nm}$ data were only acquired during the third time series. The cadence of the IMaX data was always $33 \mathrm{~s}$, while it was $12 \mathrm{~s}$ for the SuFI data of the first two time series (i.e., all recorded SuFI wavelengths within $12 \mathrm{~s}$ ) and it was $39 \mathrm{~s}$ for the last time series that included the $214 \mathrm{~nm}$ channel.

The data were corrected for dark current and flat field. Additionally, the instrumental polarization was removed from the IMaX data with the help of Mueller matrices determined by pre-flight polarimetric calibrations. Finally, the in-flight phase-diversity measurements (permanently for SuFI and intermittently for IMaX) were used to correct the images for low-order wavefront aberrations (Hirzberger et al. 2010a; Martínez Pillet et al. 2010). The phase-diversity reconstruction of the SuFI images was done using averaged wavefront errors (level3 data, see Hirzberger et al. 2010b). All intensity images were normalized to the intensity level of the mean quiet Sun, $I_{\mathrm{QS}}$, which was defined as the average of the whole image. The images were then re-sampled to the common plate scale of $0 .^{\prime \prime} 0207$ pixel $^{-1}$ (original plate scale of SuFI's $300 \mathrm{~nm}$ images) via bilinear interpolation, and the common field of view (FOV) of $13^{\prime \prime} \times 38^{\prime \prime}$ was determined. Residual noise was removed by applying a running boxcar filter of $3 \times 3$ pixels. Its width corresponds to about half of the spatial resolution of about 0 .' 15 reached by the considered observations (determined from radially averaged power spectra). As a proxy of the longitudinal magnetic field, the circular polarization degree $\left\langle p_{\text {circ }}\right\rangle$ averaged over the four points in the line $(-80,-40,+40,+80 \mathrm{~m} \AA)$,

$$
\left\langle p_{\text {circ }}\right\rangle=\frac{1}{4} \sum_{i=1}^{4} a_{i} \frac{V_{i}}{I_{i}},
$$

was calculated with a reversed sign of the two red wavelength points $(\vec{a}=[1,1,-1,-1])$ to avoid cancellation. The line-of-sight (LOS) component of the velocity vector was obtained by a Gaussian fit to the Stokes $I$ profiles. The velocity maps were corrected for the wavelength shift over the FOV caused by the IMaX etalon, and a convective blueshift of $200 \mathrm{~m} \mathrm{~s}^{-1}$ was removed (see Martínez Pillet et al. 2010).

Only one set of IMaX and SuFI observations every five minutes was analyzed in order to allow BPs to evolve between images. In total, we analyzed 19 image sets distributed over the three time series. BPs were manually identified in the $\mathrm{CN}$ images at $388 \mathrm{~nm}$, in order to be consistent with earlier work that concentrated on visible wavelengths (e.g. Zakharov et al. 2005; Berger et al. 2007). At each of the other wavelengths, we then determined the local brightness maximum in a $11 \times 11$ pixel (i.e., 0 .' $22 \times 0$ "' 22 ) environment of the detected BP position at $388 \mathrm{~nm}$. This method takes into account that the various wavelengths represent different atmospheric layers (most obvious for the Ca II H images) so that inclined magnetic features may appear at slightly different horizontal positions at different wavelengths. In total, we detected $398 \mathrm{BPs}$, of which 211 were from the third time series including the $214 \mathrm{~nm}$ band.

\section{RESULTS}

Figure 1 shows BPs at 14:27:08 UT in a region of $10^{\prime \prime} \times 8^{\prime \prime}$ (subregion of the $13^{\prime \prime} \times 38^{\prime \prime}$ full FOV). The six upper panels display the five SuFI filtergrams and the IMaX Stokes $I$ continuum image $(+227 \mathrm{m \AA})$. Most of the bright features are visible in each filtergram. However, the contrast of these features at $525 \mathrm{~nm}$ is significantly lower than in the five shorter wavelengths. Some features show a prominent brightness enhancement in the Ca II $\mathrm{H}$ image but are of moderate brightness at the other wave-

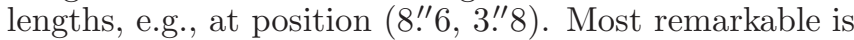
the high contrast of the BPs at $214 \mathrm{~nm}$, which strongly exceeds granulation brightness variations. It is this high contrast that lets the granulation appear relatively dark in the first panel of Figure 1, since the gray scales of all panels are adapted to the full $\mathrm{min} / \mathrm{max}$ range. The bottom left panel shows the LOS component of the velocity. While the three BPs at the positions $\left(1 !^{\prime \prime} 0,1 !^{\prime \prime} 7\right)$,

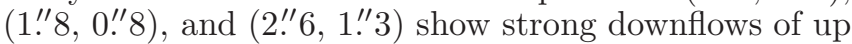
to $2.5 \mathrm{~km} \mathrm{~s}^{-1}$, most other BPs exhibit only moderate velocities. The bottom right panel displays the mean circular polarization degree. Most of the BPs in the plotted region are associated with negative polarity. Nonetheless, several bipolar regions can be seen, for instance the two neighboring BPs at $\left(2 !^{\prime \prime} 3,7 !^{\prime \prime} 3\right)$ and at $\left(2 !^{\prime} 6,7 !^{\prime \prime} 7\right)$, respectively.

Histograms of the BP peak intensity relative to the mean quiet-Sun intensity are displayed in Figure 2 as red lines in panels $(\mathrm{a})-(\mathrm{f})$. The blue lines correspond to 

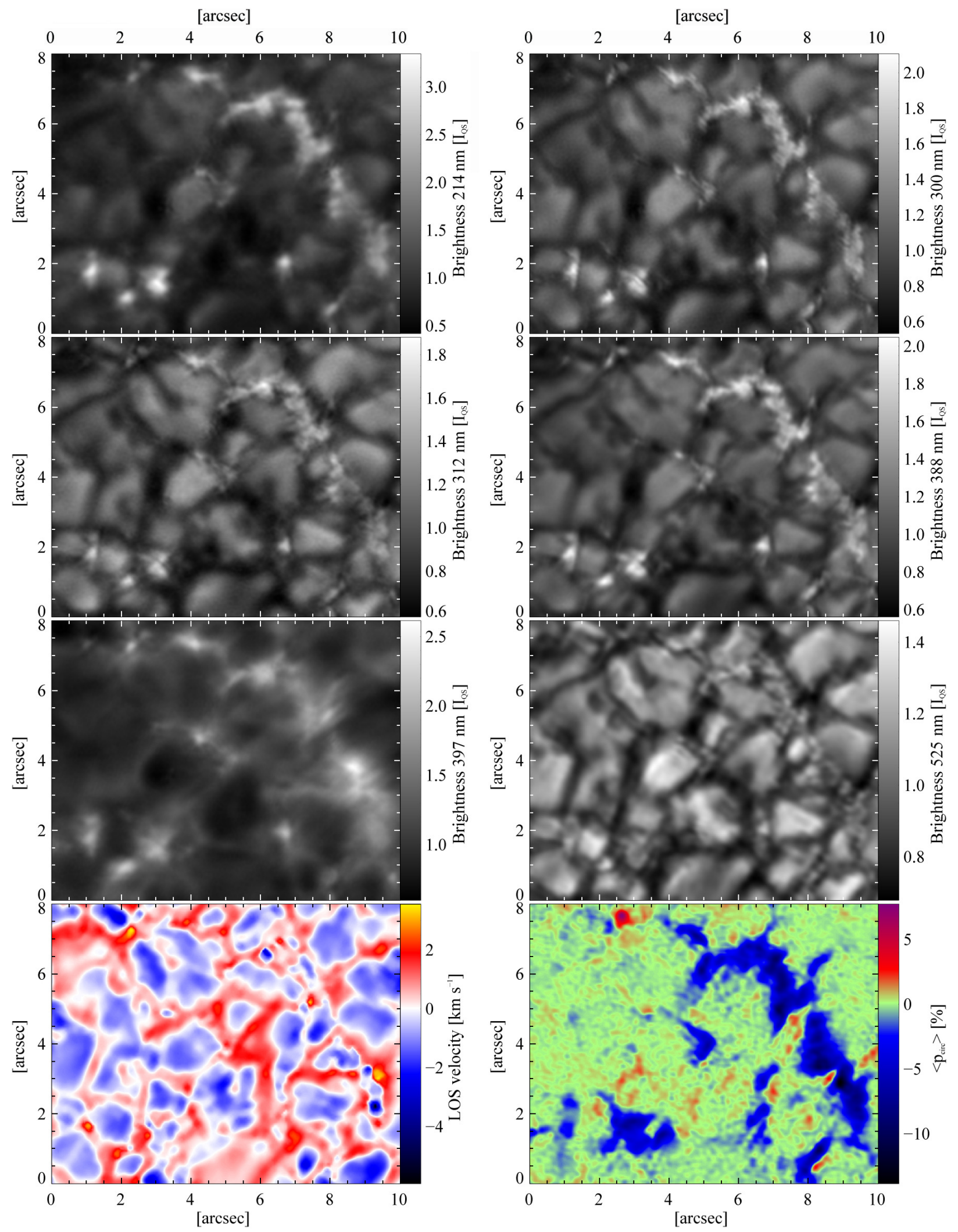

FIG. 1. - Intensity maps of the five wavelengths observed by SuFI (two first rows and the left panel of the third one) and of the continuum sample by IMaX (the right panel of the third row), all normalized to the corresponding mean intensity level of the quiet Sun, $I_{\mathrm{QS}}$. The LOS velocity obtained from a Gaussian fit (positive velocities correspond to downflows) and the mean circular polarization degree (see the main text for definition) are shown in the bottom panels. 
the brightness histograms of the darkest pixel whose distance to a BP's brightest pixel is less than $0 !^{\prime \prime} 3$ (typical width of an intergranular lane). The text labels denote the mean values. The red histograms are largely symmetric, although there is a tendency for a tail toward higher contrast values, in particular at $214 \mathrm{~nm}$ and $397 \mathrm{~nm}$. As already indicated in Figure 1, the largest average contrast is shown by BPs in the $214 \mathrm{~nm}$ image. The highest peak brightness of $5.0 I_{\mathrm{QS}}$ is also reached at this wavelength. At first sight it might be surprising that some BPs are less bright than some of the pixels of the blue histograms. A closer look reveals, however, that all BPs are indeed bright relative to the pixels in their immediate surroundings. For the CaII $\mathrm{H}$ line, the mean intensity of the darkest pixels in the BPs' vicinity is, with $1.07 I_{\mathrm{QS}}$, higher than for the other wavelengths, because the $\mathrm{Ca}$ structures are generally more diffuse and larger than our limit of $0 . / 3$. The green curves give histograms of the normalized intensity for all pixels in all frames, practically representing the intensity distribution of quiet-Sun granulation. A comparison of the maximum position of the red and green histograms clearly shows that the BPs are much more conspicuous in the UV than in the visible spectral range. The quiet-Sun histograms are more extended toward higher intensities than the histograms for the BP background, since the latter largely represents intergranular lanes. Exceptions are the histograms for CaII H, which shows reverse granulation and those for $214 \mathrm{~nm}$, where the comparable width indicates structures intermediate between reverse and normal granulation (see Solanki et al. 2010).

Panel (g) of Figure 2 shows the LOS velocity histograms for the brightest BP pixels (red), the darkest pixels of their vicinity (blue), and for all pixels (green). On average, the LOS velocity of the darkest pixels shows a downflow of $730 \mathrm{~m} \mathrm{~s}^{-1}$, while the BPs themselves are associated with a clearly weaker average downflow of $240 \mathrm{~m} \mathrm{~s}^{-1}$. However, the broad wings of the distribution contain BPs with significant upflows of up to $-3.1 \mathrm{~km} \mathrm{~s}^{-1}$ or downflows of up to $3.6 \mathrm{~km} \mathrm{~s}^{-1} \cdot 7.5 \%$ of the analyzed BPs have upflows with $v_{\text {LOS }}<-1 \mathrm{~km} \mathrm{~s}^{-1}$ and $15 \%$ of the BPs show downflows with $v_{\text {LOS }}>1 \mathrm{~km} \mathrm{~s}^{-1}$. The red histogram (BP velocities) is located between the green histogram (dominated by the large number of pixels showing upflows) and the blue histogram (mainly intergranular pixels showing downflows). Our velocities are obtained from a Gaussian fit to the Stokes $I$ profiles. The Stokes $V$ zero-crossings may possibly show different velocities if a $\mathrm{BP}$ is not spatially resolved.

In contrast to the last panel of Figure 11 where the signed net circular polarization was plotted, panel (h) of Figure 2 displays the histogram of the unsigned total polarization degree $\langle p\rangle$ averaged over the four wavelength points in the Fe I line,

$$
\langle p\rangle=\frac{1}{4} \sum_{i=1}^{4} \sqrt{\left(\frac{Q_{i}}{I_{i}}\right)^{2}+\left(\frac{U_{i}}{I_{i}}\right)^{2}+\left(\frac{V_{i}}{I_{i}}\right)^{2}} .
$$

This histogram is highly asymmetric. The polarization degree at the positions of the peak brightness reaches values of up to $9.1 \%$, the mean value is $3.24 \%$. Such large values of the polarization in $525.02 \mathrm{~nm}$ suggest that at least some of the magnetic features have been resolved
TABLE 2

Mean VAlues and Standard DEVIations of the RED HISTOGRAMS REFERRING TO THE BRIGHT POINTS (LABELED AS BP) AND BLUE HISTOGRAMS OF THE DARK BACKGROUND (LABELED AS DB) of Figure 2 The Rightmost COlumn compares the BP CONTRASTS WITH THE RMS GRANULATION CONTRAST (SEE MAIN TEXT FOR DETAILS)

\begin{tabular}{llllll}
\hline Quantity & Mean & $\sigma$ & Mean & $\sigma$ & $\begin{array}{l}\text { Contrast } \\
\text { RP }\end{array}$ \\
& BP & DB & DB & Ratio \\
\hline$\left(I / I_{\mathrm{QS}}\right)_{214}$ & 2.31 & 0.68 & 0.87 & 0.21 & 4.7 \\
$\left(I / I_{\mathrm{QS}}\right)_{300}$ & 1.52 & 0.29 & 0.81 & 0.11 & 2.4 \\
$\left(I / I_{\mathrm{QS}}\right)_{312}$ & 1.35 & 0.21 & 0.79 & 0.10 & 1.8 \\
$\left(I / I_{\mathrm{QS}}\right)_{388}$ & 1.60 & 0.25 & 0.81 & 0.11 & 3.3 \\
$\left(I / I_{\mathrm{QS}}\right)_{397}$ & 1.89 & 0.34 & 1.07 & 0.20 & 4.0 \\
$\left(I / I_{\mathrm{QS}}\right)_{525}$ & 1.11 & 0.10 & 0.81 & 0.06 & 0.8 \\
$v_{\mathrm{LOS}}\left(\mathrm{m} \mathrm{s}^{-1}\right)$ & 240 & 910 & 730 & 650 & \\
$\langle p\rangle(\%)$ & 3.24 & 1.82 & 1.40 & 0.84 & \\
\hline
\end{tabular}

(see Lagg et al. 2010, for more details). However, $4.3 \%$ of the BPs are associated with $\langle p\rangle<1 \%$. Although the polarization degree in the darkest surrounding pixels is weaker, almost all of them still show a significant polarization degree of more than $0.3 \%$ (three times the noise level) with the strongest value being 6.6\%. Polarization usually displays larger structures than the BPs (see the bottom right panel of Figure 1 and Title \& Berger 1996), which can also be concluded from the fact that the blue histogram shows larger polarization values than the green histogram for all pixels.

The mean values and their standard deviations for all red and blue histograms in Figure 2 are summarized in Table 2. Columns labeled with BP refer to histograms of the $\mathrm{BP}$ peak value, while $\mathrm{DB}$ denotes the histograms of the dark background. The standard deviation of the BP brightness is $\approx 0.5\left(\left\langle I / I_{\mathrm{QS}}\right\rangle-1\right)$ in all the SuFI channels, except Ca II H. This relationship can be used to estimate the brightness histogram width for other wavelengths. The rightmost column of the table shows the ratio of the $\mathrm{BP}$ contrast (mean BP brightness value minus one) to the rms intensity contrast (taken from Hirzberger et al. 2010b, and calculated over the whole FOV of the level-3 data). This contrast is a measure of how strongly BPs stand out relative to the surrounding granulation (or reversed granulation and waves in the case of $397 \mathrm{~nm}$ ). According to Table 2] images at $214 \mathrm{~nm}$ show the BPs most clearly.

\section{SUMMARY AND DISCUSSION}

We identified 398 BPs in simultaneously observed photometric and polarimetric SUNRISE images of a quietSun region close to the disk center. Our data include three wavelengths in the near-UV in which the Sun was never observed before at high spatial resolution. We determined the peak brightness and the brightness of the dark background of every detected BP at each observed wavelength. The BPs' peak intensity reaches up to 5.0 times the mean quiet-Sun intensity $I_{\mathrm{QS}}$ at $214 \mathrm{~nm}$. The mean peak intensity at that wavelength is $2.31 I_{\mathrm{QS}}$. The $214 \mathrm{~nm}$ wavelength also displays the largest ratio of BP contrast to the rms of the intensity over the whole FOV (see the rightmost column of Table 2). This ratio is a measure of how prominent BPs are in an image at a particular wavelength. These values indicate that they are even more prominent at $214 \mathrm{~nm}$ than in the core of 

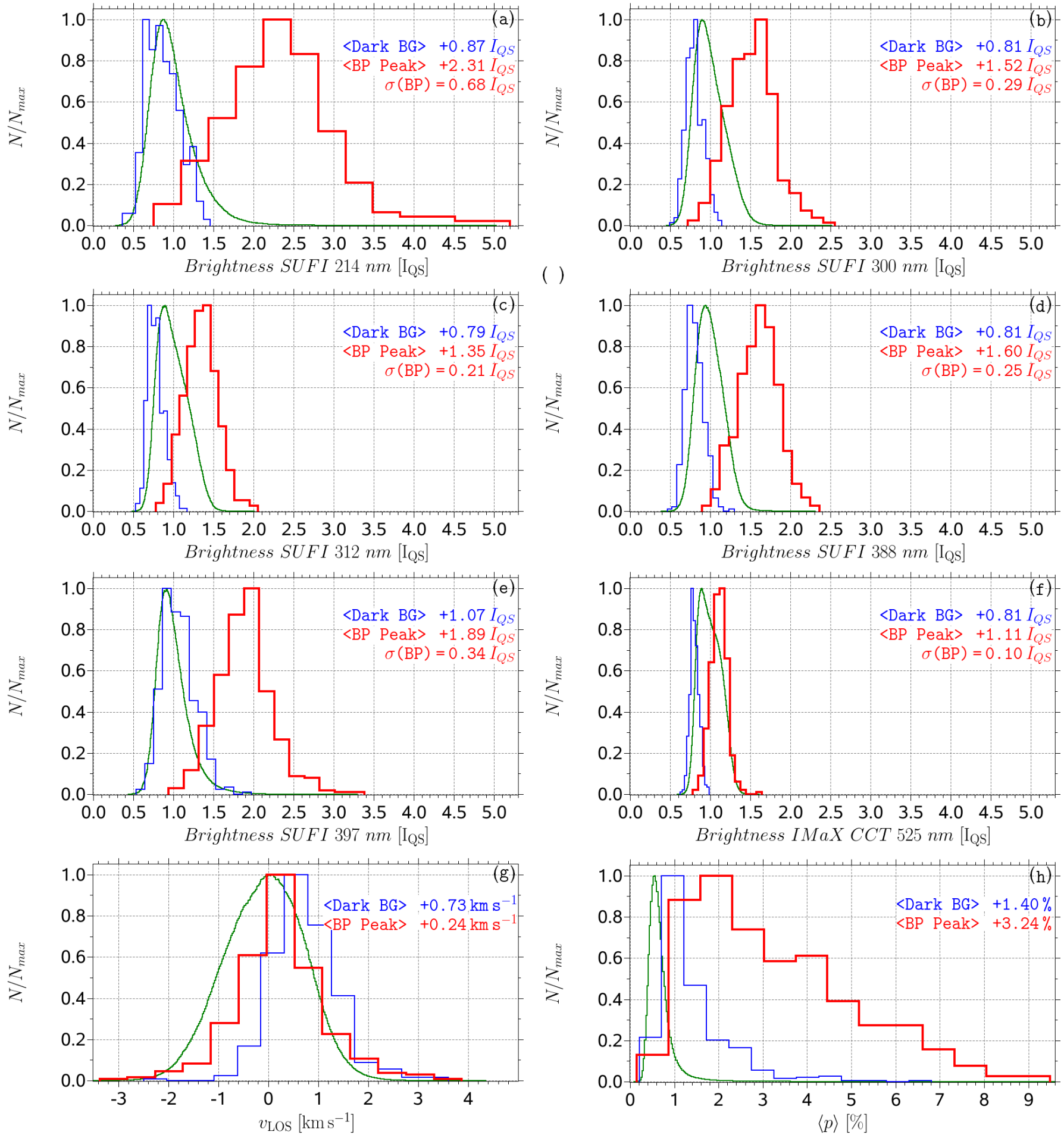

FIG. 2.- Brightness histograms of the six wavelengths observed by SunRISE together with histograms of the LOS velocity and the averaged polarization degree. Red lines correspond to histograms of the BPs' brightest pixel, blue lines to the darkest pixel in a $0 .{ }^{\prime \prime} 3$ vicinity of the bright point, and the green lines denote histograms of all pixels in all frames. The mean values of the red and blue histograms as well as the standard deviations of the red brightness histograms are indicated as the text labels. 
Ca II H (for the $0.18 \mathrm{~nm}$ wide filter employed by SuFI).

The value of the mean peak brightness at $388 \mathrm{~nm}$ (1.60 $I_{\mathrm{QS}}$ ) agrees exactly with the $\mathrm{CN}$ peak brightness obtained by Zakharov et al. (2005) from data recorded with the $1 \mathrm{~m}$ Swedish Solar Telescope (SST). Examples of BP brightness values (close to the disk center) given in the literature for the frequently observed $G$-band at $430 \mathrm{~nm}$ ( $\mathrm{CH}$ molecule) are $1.45 I_{\mathrm{QS}}$ (Zakharov et al. 2005) and 1.2 I $\mathrm{QS}$ (Berger et al.|2007). Both studies analyzed SST data. Utz et al. (2009) used $G$-band data from the $50 \mathrm{~cm}$ Solar Optical Telescope onboard Hinode and found $1.3 I_{\mathrm{QS}}$. These values are comparable with what we find in the near-UV, with the exception of $214 \mathrm{~nm}$, which displays distinctly higher values. Note that the BP contrasts given in this study are not corrected for instrumental scattered light. After this correction, the BP contrasts will most likely increase (see, e.g., Wedemever-Böhm 2008; Mathew et al. 2009, A. Feller et al. 2010, in preparation).

From the spectropolarimetric data we derived the LOS component of the velocity vector. The largest $\mathrm{BP}$ velocities reach $-3.1 \mathrm{~km} \mathrm{~s}^{-1}$ in upflows and $3.6 \mathrm{~km} \mathrm{~s}^{-1}$ in downflows. The mean value is $240 \mathrm{~m} \mathrm{~s}^{-1}$, in good agreement with the average velocity of $260 \mathrm{~m} \mathrm{~s}^{-1}$ published by Beck et al. (2007) and reasonably consistent with the absence of Stokes $V$ zero-crossing shifts found by Solanki (1986) and Martínez Pillet et al. (1997) in data with much lower spatial resolution. In contrast to this, Grossmann-Doerth et al. (1996) reported a stronger mean downflow of $800 \mathrm{~m} \mathrm{~s}^{-1}$ as derived from the zerocrossing of their Stokes $V$ profiles and Sigwarth et al. (1999) found a velocity range of $\pm 5 \mathrm{~km} \mathrm{~s}^{-1}$ and a mean velocity of $500 \mathrm{~m} \mathrm{~s}^{-1}$. The nature of the BPs displaying strong up- or downflows in Stokes $I$ will be investigated in a subsequent study.

The polarization degree, averaged over the four points within the FeI line, is also calculated from the IMaX data and shows values up to $9.1 \%$. The mean BP polarization degree is $3.24 \%$, which is clearly above the mean signal of $1.40 \%$ for the dark vicinity. Intriguingly, about $4 \%$ of the BPs are associated with relatively weak magnetic flux $(\langle p\rangle<1 \%)$. This raises interesting questions, since enhanced temperatures and hence brightness in magnetic elements (flux tubes) is caused by evacuation, which in turn is proportional to $B^{2}$. Therefore, we would expect that BPs show strong Stokes $V$ sig- nals. Significantly inclined magnetic fields cannot explain the observed weak Stokes $V$ signals, because we also measured weak Stokes $Q$ and $U$ signals. Additionally, strongly evacuated flux tubes are strongly buoyant and hence should be nearly vertical (Schüssler 1986). Lagg et al. (2010) find a strong Stokes $I$ line weakening for kilo-Gauss network patches due to temperature enhancements in the flux tubes. Such an absorption weakening can also lead to a weak Stokes $V$ signal. Although the network patches analyzed by Lagg et al. (2010) are spatially resolved, we find many BPs that exhibit complex Stokes profiles, which is a clear indication that not all of the BPs are spatially resolved. Insufficient resolution can also contribute to weak Stokes $V$ signals.

In summary, we find high intensity contrasts of BPs in the near-UV range (including the first measurements below $388 \mathrm{~nm}$ ), with values up to $5 I_{\mathrm{QS}}$ at $214 \mathrm{~nm}$. The simultaneous spectropolarimetric measurements confirm the close association of BPs with magnetic flux concentrations in intergranular downflow lanes. However, the majority of the BPs exhibit only weak downflows.

The reasonably high cadence of SunRISE data (between $4 \mathrm{~s}$ and $39 \mathrm{~s}$, depending on the number of observed wavelengths and their exposure times) and the high measured contrasts of BPs make detailed future studies of the dynamical properties of BPs very promising (e.g., S. Jafarzadeh et al. 2010, in preparation). Of considerable additional benefit for such studies will be the possibility to compare the dynamics at different layers in the solar atmosphere, which are covered by the combination of SuFI and IMaX wavelength bands.

The German contribution to SunRISE is funded by the Bundesministerium für Wirtschaft und Technologie through Deutsches Zentrum für Luft- und Raumfahrt e.V. (DLR), Grant No. 50 OU 0401, and by the Innovationsfond of the President of the Max Planck Society (MPG). The Spanish contribution has been funded by the Spanish MICINN under projects ESP200613030-C06 and AYA2009-14105-C06 (including European FEDER funds). The HAO contribution was partly funded through NASA grant number NNX08AH38G. This work has been partly supported by the WCU grant (No. R31-10016) funded by the Korean Ministry of Education, Science \& Technology.

\section{REFERENCES}

Barthol, P., et al. 2010, Sol. Phys., in press (arXiv:1009.2689)

Beck, C., Bellot Rubio, L. R., Schlichenmaier, R., \& Sütterlin, P. 2007, A\&A, 472, 607

Berger, T. E., Rouppe van der Voort, L., \& Löfdahl, M. 2007, ApJ, 661, 1272

Berger, T. E., Schrijver, C. J., Shine, R. A., Tarbell, T. D., Title, A. M., \& Sharmer, G. 1995, ApJ, 454, 531

Berkefeld, T., et al. 2010, Sol. Phys., in press arXiv:1009.3196

Deinzer, W., Hensler, G., Schüssler, M., \& Weisshaar, E. 1984, A\&A, 139, 426

Dunn, R. B., \& Zirker, J. B. 1973, Sol. Phys., 33, 281

Gandorfer, A., et al. 2010, Sol. Phys., in press (arXiv:1009.1037)

Grossmann-Doerth, U., Keller, C. U., \& Schüssler, M. 1996, A\&A, 315, 610

Hirzberger, J., et al. 2010a, A\&A, submitted

Hirzberger, J., et al. 2010b, ApJ, this issue

Krivova, N. A., Solanki, S. K., Fligge, M., \& Unruh, Y. C. 2003, A\&A, 399, L1
Krivova, N. A., Solanki, S. K., \& Floyd, L. 2006, A\&A, 452, 631

Lagg, A., et al. 2010, ApJ, this issue

Martínez Pillet, V., Lites, B. W., \& Skumanich, A. 1997, ApJ, 474,810

Martínez Pillet, V., et al. 2010, Sol. Phys., in press (arXiv:1009.1095)

Mathew, S. K., Zakharov, V., \& Solanki, S. K. 2009, A\&A, 501, L19

Mehltretter, J. P. 1974, Sol. Phys., 38, 43

Muller, R., \& Roudier, Th. 1984, Sol. Phys., 94, 33

Schüssler, M. 1986, Small Scale Magnetic Flux Concentrations in the Solar Photosphere, ed. W. Deinzer, M. Knölker, \& H. H.

Voigt (Göttingen: Vandenhoeck \& Ruprecht), 103

Sigwarth, M., Balasubramaniam, K. S., Knölker, M., \& Schmidt, W. 1999, A\&A, 349, 941

Solanki, S. K. 1986, A\&A, 168, 311

Solanki, S. K. 1993, Space Sci. Rev., 63, 1

Solanki, S. K., \& Fligge, M. 2002, Adv. Space Res., 29, 1933 
Solanki, S. K., et al. 2010, ApJ, this issue

Spruit, H. C. 1976, Sol. Phys., 50, 269

Title, A. M., \& Berger, T. E. 1996, ApJ, 597, L173

Utz, D., Hanslmeier, A., Möstl, C., Muller, R., Veronig, A., \& Muthsam, H. 2009, A\&A, 498, 289

Wedemeyer-Böhm, S. 2008, A\&A, 487, 399
Wenzler, T., Solanki, S. K., Krivova, N. A., \& Fröhlich, C. 2006, A\&A, 460, 583

Zakharov, V., Gandorfer, A., Solanki, S. K., \& Löfdahl, M. 2005, A\&A, 437, L43 